\title{
Operations Preserving Global Rigidity of Generic Direction-Length Frameworks
}

\author{
Bill Jackson*and Tibor Jordán ${ }^{\dagger}$
}

September 10, 2009

\begin{abstract}
A two-dimensional direction-length framework is a pair $(G, p)$, where $G=(V ; D, L)$ is a graph whose edges are labeled as 'direction' or 'length' edges, and a map $p$ from $V$ to $\mathbb{R}^{2}$. The label of an edge $u v$ represents a direction or length constraint between $p(u)$ and $p(v)$. The framework $(G, p)$ is called globally rigid if every other framework $(G, q)$ in which the direction or length between the endvertices of corresponding edges is the same, is 'congruent' to $(G, p)$, i.e. it can be obtained from $(G, p)$ by a translation and, possibly, a dilation by -1 .

We show that labeled versions of the two Henneberg operations (0extension and 1-extension) preserve global rigidity of generic directionlength frameworks. These results, together with appropriate inductive constructions, can be used to verify global rigidity of special families of generic direction-length frameworks.
\end{abstract}

\section{Introduction}

Consider a point configuration $p_{1}, p_{2}, \ldots, p_{n}$ in $\mathbb{R}^{d}$ together with a set of constraints which fix the direction or the length between some pairs $p_{i}, p_{j}$. A basic question is whether the configuration, with the given constraints, is locally or globally unique, up to 'congruence'. Results of this type have

\footnotetext{
*School of Mathematical Sciences, Queen Mary, University of London, Mile End Road, London E1 4NS, England. e-mail: b.jackson@qmul.ac.uk. This work was supported by an International Joint Project grant from the Royal Society.

${ }^{\dagger}$ Department of Operations Research, Eötvös University, Pázmány Péter sétány 1/C, 1117 Budapest, Hungary. e-mail: jordan@cs.elte.hu. Supported by the MTA-ELTE Egerváry Research Group on Combinatorial Optimization and the Hungarian Scientific Research Fund grant no. T49671, T60802.
} 

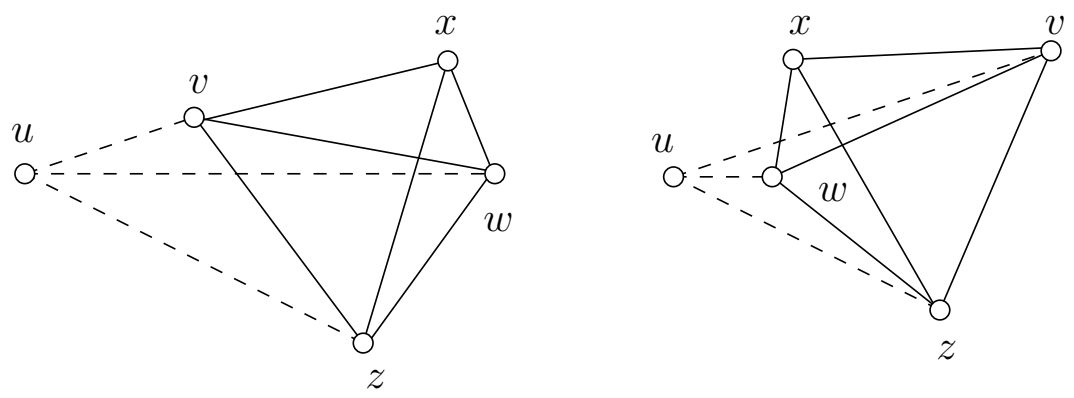

Figure 1: Two equivalent but non-congruent realizations of a mixed graph. We use solid or dashed lines to indicate edges with length or direction labels, respectively. The frameworks are not globally rigid.

applications in localization problems of sensor networks, CAD, and molecular conformation $[4,14,18]$. The configuration and the constraints form a 'direction-length framework'.

A mixed graph $G=(V ; D, L)$ is an undirected graph together with a labeling (or bipartition) $D \cup L$ of its edge set. We refer to edges in $D$ as direction edges and edges in $L$ as length edges. A $d$-dimensional directionlength framework, or more simply mixed framework, is a pair $(G, p)$, where $G=(V ; D, L)$ is a mixed graph and $p$ is a map from $V$ to $\mathbb{R}^{d}$. When $L=\emptyset$, or $D=\emptyset$, we say that $(G, p)$ is a direction framework or length framework, respectively, or simply that $(G, p)$ is a pure framework. We also say that $(G, p)$ is a realization of $G$ in $\mathbb{R}^{d}$. Two frameworks $(G, p)$ and $(G, q)$ are equivalent if: (i) $p(u)-p(v)$ is a scalar multiple of $q(u)-q(v)$ for all $u v \in D$ with $q(u) \neq q(v)$; (ii) $\|p(u)-p(v)\|=\|q(u)-q(v)\|$ for all $u v \in L$, where $\|$.$\| denotes the Euclidean norm in \mathbb{R}^{d}$.

The mixed frameworks $(G, p)$ and $(G, q)$ are congruent if $p(u)-p(v)$ is a scalar multiple of $q(u)-q(v)$ for all $u, v \in V$ with $q(u) \neq q(v)$, and $\|p(u)-p(v)\|=\|q(u)-q(v)\|$ for all $u, v \in V$. A mixed framework $(G, p)$ is globally rigid if every framework which is equivalent to $(G, p)$ is congruent to $(G, p)$. See Figure 1 .

A mixed framework $(G, p)$ is rigid if there exists an $\epsilon>0$ such that every framework $(G, q)$ which is equivalent to $(G, p)$ and satisfies $\|p(v)-q(v)\|<\epsilon$ for all $v \in V$, is congruent to $(G, p)$. This is the same as saying that every continuous motion of the points $p(v), v \in V$, which preserves the distances between pairs of points joined by length edges and directions between pairs joined by direction edges, results in a framework which is congruent to $(G, p)$.

(Global) direction rigidity and length rigidity of pure frameworks are 
defined analogously.

It is a hard problem to decide if a given framework is rigid or globally rigid. Indeed Saxe [13] has shown that the global rigidity problem is NPhard even for 1-dimensional length frameworks. The problem becomes more tractable, however, if we assume that there are no algebraic dependencies between the coordinates of the points of the framework. A framework $(G, p)$ is said to be generic if the set containing the coordinates of all its points is algebraically independent over the rationals. We say that a mixed graph $G$ is (globally) rigid in $\mathbb{R}^{d}$ if all generic realizations of $G$ in $\mathbb{R}^{d}$ are (globally) rigid. The underlying mixed graph of the frameworks in Figure 1 is rigid but not globally rigid in $\mathbb{R}^{2}$.

(Global) length rigidity and direction rigidity of graphs are defined analogously.

Whiteley [17] showed that a graph is direction rigid in $\mathbb{R}^{d}$ if and only if it is globally direction rigid in $\mathbb{R}^{d}$ and characterized the graphs which have this property. In contrast, length rigidity of graphs in $\mathbb{R}^{d}$ is a weaker property than global length rigidity for all $d \geq 1$, and graphs with these properties have been characterized only for $d=1,2$. The cases when $d=1$ are not difficult. The characterizations of length rigidity and global length rigidity when $d=2$ are given in [11] and [8], respectively.

Henceforth, we assume that $d=2$ unless specified otherwise. The characterizations of (global) direction and length rigidity in this case can be formulated as inductive constructions using the following graph operations. The operation 0 -extension on vertices $u, w$ of a graph $G$ adds a new vertex $v$ and new edges $v u, v w$. The operation 1-extension on edge $u w$ and vertex $t$ of $G$ deletes the edge $u w$ and adds a new vertex $v$ and new edges $v u, v w, v t$. See Figures 2, 3. These operations, which are sometimes called vertex addition and edge splitting, respectively, are known as Henneberg operations since they were first used in the study of rigidity by Henneberg [7]. Parts (a) and (b) of the next theorem follow from results of Whiteley [17] and Tay and Whiteley [16]. Part (c) is from [8].

Theorem 1.1 Let $G$ be a graph. Then

(a) $G$ is (globally) direction rigid if and only if $G$ can be obtained from $K_{2}$ by 0-extensions, 1-extensions, and edge-additions.

(b) $G$ is length rigid if and only if $G$ can be obtained from $K_{2}$ by 0 -extensions, 1-extensions, and edge-additions.

(c) $G$ is globally length rigid if and only if $G=K_{2}, G=K_{3}$ or $G$ can be obtained from $K_{4}$ by 1-extensions and edge-additions.

Servatius and Whiteley [14] used mixed versions of the 0- and 1-extension 


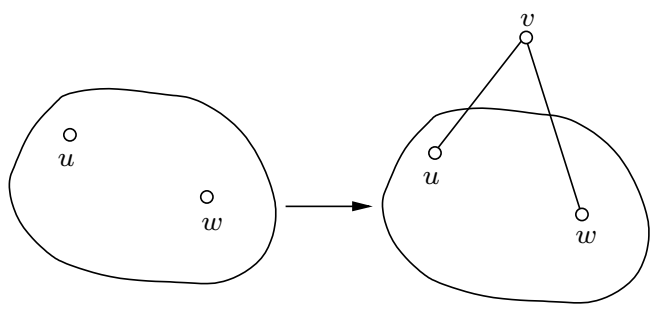

Figure 2: The 0-extension operation.

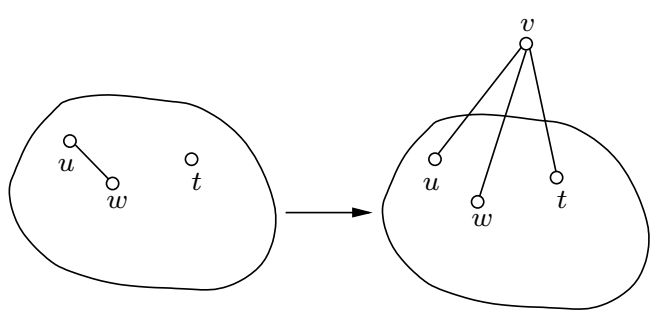

Figure 3: The 1-extension operation.

operations to characterize rigid mixed graphs. These operations are defined as follows. A 0-extension on vertices $u, w$ of a mixed graph $G$, adds a new vertex $v$ and new edges $v u, v w$ in such a way that if $v u, v w$ are of the same type (i.e. either they are both length edges or both direction edges) then $u, w$ must be distinct. A 1-extension on edge uw and vertex $t$ of $G$, deletes an edge $u w$ and adds a new vertex $v$ and new edges $v u, v w, v t$ for some vertex $t \in V(G)$, with the provisos that at least one of the new edges has the same type as the deleted edge and, if $t=u$, then the two edges from $v$ to $u$ are of different type. Servatius and Whiteley's results give rise to the following inductive construction for rigid mixed graphs.

Theorem 1.2 [14] Let $G$ be a mixed graph. Then $G$ is rigid if and only if $G$ can be obtained from the mixed graph with two vertices joined by both a length edge and a direction edge by 0-extensions, 1-extensions, and edge-additions.

Direction or length rigid graphs as well as rigid mixed graphs can also be characterized by 'counts', i.e. the existence of certain sparse subgraphs (see for example [10]).

The problem of characterizing when a mixed graph $G$ is globally rigid is still an open problem. To solve this problem using a similar strategy to that for global length rigidity, we would require results which assert that 
Henneberg operations preserve global rigidity in mixed graphs, as well as an inductive construction for the conjectured family of globally rigid mixed graphs which uses these operations. In this paper we present results of the first type by showing that mixed versions of the Henneberg operations preserve global rigidity:

Theorem 1.3 Let $G$ and $H$ be mixed graphs with $|V(H)| \geq 2$. Suppose that $G$ can be obtained from $H$ by a 0 -extension which adds a vertex $v$ incident to two direction edges. Then $G$ is globally rigid if and only if $H$ is globally rigid.

Theorem 1.4 Let $G$ and $H$ be mixed graphs with $|V(H)| \geq 3$. Suppose that $G$ can be obtained from $H$ by a 1-extension on an edge uw. Suppose further that $H$ is globally rigid and $H-u w$ is rigid. Then $G$ is globally rigid.

A result on globally length rigid graphs analogous to Theorem 1.4 is given in [9], see also [3].

A mixed graph $G$ is redundantly rigid if $G-e$ is rigid for all edges $e$ of $G$. The above mentioned characterization of rigid mixed graphs, and in particular Theorem 1.2, imply that every 1-extension of a redundantly rigid mixed graph is redundantly rigid. We may combine this with Theorem 1.4 to obtain

Theorem 1.5 Let $G$ and $H$ be mixed graphs. Suppose that $H$ is globally rigid and redundantly rigid, and that $G$ can be obtained from $H$ by a sequence of 1-extensions. Then $G$ is globally rigid.

\section{An application of our results}

We use Theorems 1.3 and 1.4 in our companion paper [10], together with new inductive constructions, to characterize globally rigidity for the special family of graphs $G=(V ; D, L)$ which are redundantly rigid and have $|D|+$ $|L|=2|V|-1$. Such graphs are called mixed circuits since their edges sets are circuits in the direction-length rigidity matroid (which will be defined in the next section). This characterization complements the results on globally length rigid graphs whose edge set is a circuit in the length rigidity matroid [2] and may serve as a building block in a more complete characterization of mixed global rigidity. The two smallest mixed circuits are shown in Figure 4.

To state the characterization we need some more definitions (that we shall also need in Section 5). A 2-separation of $G$ is a pair of subgraphs 

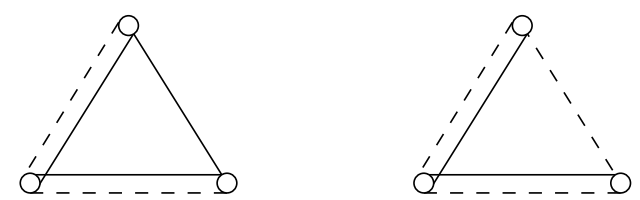

Figure 4: The two mixed circuits on three vertices. These graphs, denoted by $K_{3}^{+}$and $K_{3}^{-}$, are the smallest (mixed) circuits of the direction-length rigidity matroid.

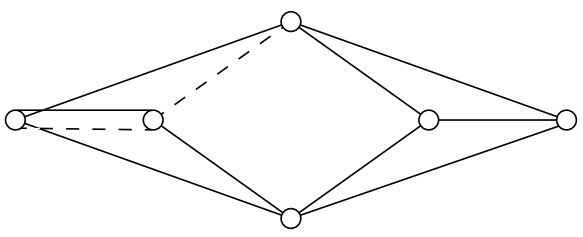

Figure 5: A mixed graph $G$ for which no generic realization is globally rigid. This follows from the fact that one side of a 2-separation of $G$ contains length edges only and hence can be reflected in the line through the two common vertices in the 2 -separation.

$G_{1}, G_{2}$ such that $G=G_{1} \cup G_{2},\left|V\left(G_{1}\right) \cap V\left(G_{2}\right)\right|=2$ and $V\left(G_{1}\right)-V\left(G_{2}\right) \neq$ $\emptyset \neq V\left(G_{2}\right)-V\left(G_{1}\right)$. We refer to the pair of common vertices of $G_{1}$ and $G_{2}$ as a 2-separator of $G$. We say that a 2 -separation $\left(G_{1}, G_{2}\right)$ of $G$ is directionbalanced if both $G_{1}$ and $G_{2}$ contain an edge in $D$. A 2-separation which is not direction-balanced is said to be direction-unbalanced. A mixed graph is direction-balanced if all its 2-separations are direction-balanced. It is easy to see that if $G$ is globally rigid then $G$ is direction balanced [10, Lemma 1.6], see Figure 5 .

We will also need one further operation on mixed graphs. Suppose that $G_{1}=\left(V_{1}, E_{1}\right)$ and $G_{2}=\left(V_{2}, E_{2}\right)$ are graphs with $V_{1} \cap V_{2}=\{u, v\}$ and $E_{1} \cap E_{2}=\{u v\}$. Then we say that the graph $G=\left(G_{1}-u v\right) \cup\left(G_{2}-u v\right)$ is a 2 -sum of $G_{1}$ and $G_{2}$, and write $G=G_{1} \oplus_{2} G_{2}$. When $G_{i}=\left(V_{i} ; D_{i}, L_{i}\right)$ is a mixed graph for each $i \in\{1,2\}$ and $u v$ has the same type in both $G_{1}$ and $G_{2}$, their 2-sum is the mixed graph $\left(V_{1} \cup V_{2} ;\left(D_{1} \cup D_{2}\right)-\{u v\},\left(L_{1} \cup L_{2}\right)-\{u v\}\right)$. The mixed graph in Figure 5 is an example of a 2 -sum.

The main result of [10] is an inductive construction: we show that a mixed graph is a direction balanced mixed circuit if and only if it can be obtained from $K_{3}^{+}$or $K_{3}^{-}$by a sequence of 1-extensions and 2-sums with direction-pure $K_{4}$ 's $[10$, Theorem 5.10]. Note that a 2 -sum with a directionpure $K_{4}$ can also be obtained by a 0 -extension, which adds two direction 


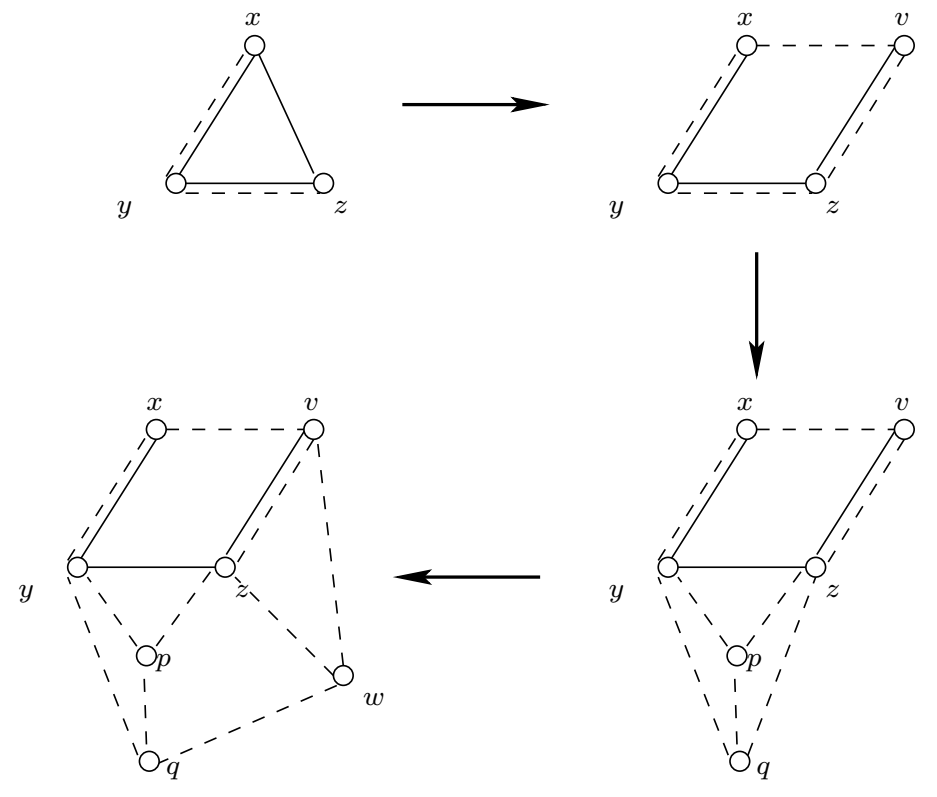

Figure 6: The inductive construction of a direction balanced (and hence globally rigid) mixed circuit. The graph is obtained from $K_{3}^{+}$by a $1-$ extension (adding vertex $v$ ), followed by a 2 -sum with a direction-pure $K_{4}$ (on vertex set $\{y, z, p, q\}$ ) and then another 1-extension which adds $w$.

edges, followed by a 1-extension. The construction is illustrated by Figure 6.

Combining Theorems 1.3 and 1.4 of this paper with this inductive construction we obtain:

Theorem 1.6 [10, Theorem 6.2] Let $G$ be a mixed circuit. Then $G$ is globally rigid if and only if $G$ is direction balanced.

We close this section with an outline of the paper. In Section 2 we will show, for completeness, that infinitesimal rigidity (defined in the next section) is a sufficient condition for rigidity in mixed frameworks, and that the two conditions are equivalent for generic mixed frameworks. The above mentioned characterization of rigid mixed graphs is given in terms of infinitesimal rigidity in [14] and we need this equivalence to justify our statement of their result, Theorem 1.2. In Section 3 we introduce quasi-generic frameworks and derive a result about the field extension $\mathbb{Q}(p)$ of a quasigeneric rigid framework $(G, p)$ which will be key to our main results on 
extensions. For convenience, some proofs from Sections 2 and 3 are deferred to the Appendix. Section 4 contains the proofs of the main results on 0and 1-extensions. We also provide further applications of our methods in the study of continuous motions of generic mixed frameworks and globally linked pairs in mixed circuits in Sections 3 and 5, respectively. Section 6 is devoted to some concluding remarks on the problem of characterizing globally rigid mixed graphs.

\section{Infinitesimal rigidity and the rigidity matrix}

Servatius and Whiteley [14] developed a rigidity theory for mixed frameworks analogous to that given for pure frameworks. For $(x, y) \in \mathbb{R}^{2}$ let $(x, y)^{\perp}=(y,-x)$. Note that we take $(0,0)^{\perp}=(0,0)$. The rigidity matrix of a mixed framework $(G, p)$ is the matrix $R(G, p)$ of size $(|D|+|L|) \times 2|V|$, where, for each edge $u v \in D \cup L$, in the row corresponding to $u v$, the entries in the two columns corresponding to the vertex $w$ are given by: $(p(u)-p(v))^{\perp}$ if $u v \in D$ and $w=u ;-(p(u)-p(v))^{\perp}$ if $u v \in D$ and $w=v ;(p(u)-p(v))$ if $u v \in L$ and $w=u ;-(p(u)-p(v))$ if $u v \in L$ and $w=v ;(0,0)$ if $w \notin\{u, v\}$. The rigidity matrix of $(G, p)$ defines the rigidity matroid of $(G, p)$ on the ground set $D \cup L$ by linear independence of the rows of the rigidity matrix. The framework is said to be independent if the rows of $R(G, p)$ are linearly independent. Since the vectors $(1,0,1,0, \ldots, 1,0)$ and $(0,1,0,1, \ldots, 0,1)$ belong to the null space of $R(G, p)$, rank $R(G, p) \leq 2|V|-2$, and rank $R(G, p)$ remains unchanged if we delete the two columns indexed by any vertex of $G$. The framework $(G, p)$ is infinitesimally rigid if the rank of $R(G, p)$ is equal to $2|V|-2$. We will show that infinitesimal rigidity is a sufficient condition for rigidity in any mixed framework and that the two concepts are equivalent for generic mixed frameworks.

Theorem 2.1 Suppose that $(G, p)$ is a mixed framework. If $(G, p)$ is infinitesimally rigid then $(G, p)$ is rigid. Furthermore, when $(G, p)$ is generic, $(G, p)$ is infinitesimally rigid if and only if $(G, p)$ is rigid.

Our proof of Theorem 2.1 uses elementary differential geometry and is given in Appendix A. It is similar to an analogous result for length frameworks due to Asimow and Roth [1].

Any two generic frameworks $(G, p)$ and $(G, q)$ have the same rigidity matroid. We call this the (2-dimensional) rigidity matroid $\mathcal{R}(G)$ of the mixed graph $G$. We denote the rank of $\mathcal{R}(G)$ by $r(G)$. The mixed graph $G$ is independent if $r(G)=|D|+|L|$. Independent mixed graphs were 
characterized in [14]. This gives a characterization of the rigidity matroid of a mixed graph. Theorem 2.1 implies that a mixed graph $G$ is rigid if and only if $r(G)=2|V|-2$.

\section{Quasi-generic frameworks}

A mixed framework $(G, p)$ is quasi-generic if it is a translation of a generic framework. We will be mostly concerned with quasi-generic frameworks in which one vertex is positioned at the origin. Such frameworks are characterized by the following elementary lemma.

Lemma 3.1 Let $(G, p)$ be a framework with vertices $\left\{v_{1}, v_{2}, \ldots, v_{n}\right\}, p\left(v_{1}\right)=$ $(0,0)$ and $p\left(v_{i}\right)=\left(p_{2 i-1}, p_{2 i}\right)$ for $2 \leq i \leq n$. Then $(G, p)$ is quasi-generic if and only if $\left\{p_{3}, p_{4}, \ldots, p_{2 n}\right\}$ is algebraically independent over $\mathbb{Q}$.

Our goal in this section is to show that if $(G, p)$ is a quasi-generic rigid framework, $(G, q)$ is equivalent to $(G, p)$, and $p(v)=(0,0)=q(v)$ for some vertex $v$ of $G$, then the field extensions $\mathbb{Q}(p)$ and $\mathbb{Q}(q)$ have the same algebraic closure. This will be a key fact in our proof that extensions preserve global rigidity.

Let $(G, p)$ be a mixed framework, where $G=(V ; D, L)$. For $v_{1}, v_{2} \in V$ with $p\left(v_{i}\right)=\left(x_{i}, y_{i}\right)$ let $l_{p}\left(v_{1}, v_{2}\right)=\left(x_{1}-x_{2}\right)^{2}+\left(y_{1}-y_{2}\right)^{2}$, and $s_{p}\left(v_{1}, v_{2}\right)=$ $\left(y_{1}-y_{2}\right) /\left(x_{1}-x_{2}\right)$ whenever $x_{1} \neq x_{2}$. Suppose $e=v_{1} v_{2} \in D \cup L$. We say that $e$ is vertical in $(G, p)$ if $x_{1}=x_{2}$. The length of $e$ in $(G, p)$ is given by $l_{p}(e)=l_{p}\left(v_{1}, v_{2}\right)$, and the slope of $e$ by $s_{p}(e)=s_{p}\left(v_{1}, v_{2}\right)$ whenever $e$ is not vertical in $(G, p)$.

Let $V=\left\{v_{1}, v_{2}, \ldots, v_{n}\right\}$ and $D \cup L=\left\{e_{1}, e_{2}, \ldots, e_{m}\right\}$. We view $p$ as a point $\left(p\left(v_{1}\right), p\left(v_{2}\right), \ldots, p\left(v_{n}\right)\right)$ in $\mathbb{R}^{2 n}$. Let $T$ be the set of all points $p \in \mathbb{R}^{2 n}$ such that $(G, p)$ has no vertical direction edges. Then the rigidity map $f_{G}$ : $T \rightarrow \mathbb{R}^{m}$ is given by $f_{G}(p)=\left(h\left(e_{1}\right), h\left(e_{2}\right), \ldots, h\left(e_{m}\right)\right)$, where $h\left(e_{i}\right)=l_{p}\left(e_{i}\right)$ if $e_{i} \in L$ and $h\left(e_{i}\right)=s_{p}\left(e_{i}\right)$ if $e_{i} \in D$. The rigidity map is related to the rigidity matrix $R(G, p)$ by the fact that each row in the evaluation of the Jacobian of the rigidity map at the point $p \in T,\left.d f_{G}\right|_{p}$, is a non-zero scalar multiple of the corresponding row of $R(G, p)$, and hence rank $\left.d f_{G}\right|_{p}=\operatorname{rank} R(G, p)$.

The proofs of our first two lemmas use some elementary algebraic geometry and are given in Appendix B. They are analogous to results for length frameworks given in [9].

Lemma 3.2 Suppose that $G=(V ; D, L)$ is an independent mixed graph and $(G, p)$ is a quasi-generic realization of $G$. Then $f_{G}(p)$ is generic. 
Given a point $p \in \mathbb{R}^{n}$ we use $\mathbb{Q}(p)$ to denote the field extension of $\mathbb{Q}$ by the coordinates of $p$. Given fields $K, L$ with $K \subseteq L$ the transcendence degree of $L$ over $K, t d[L: K]$, is the size of the largest subset of $L$ which is algebraically independent over $K$, see [5]. We use $\tilde{K}$ to denote the algebraic closure of $K$. Note that $t d[\tilde{K}: K]=0$.

A mixed graph $G=(V ; D, L)$ is minimally rigid if it is both rigid and independent (and hence satisfies $|D|+|L|=2|V|-2$ ).

Lemma 3.3 Let $G$ be a minimally rigid mixed graph and $(G, p)$ be a realization of $G$ with no vertical edges and with $p=\left(0,0, p_{3}, p_{4}, p_{5}, \ldots, p_{2 n}\right)$. Suppose that $f_{G}(p)$ is generic and let $L=\mathbb{Q}(p)$ and $K=\mathbb{Q}\left(f_{G}(p)\right)$. Then $\left(p_{3}, p_{4}, p_{5}, \ldots, p_{2 n}\right)$ is generic and $\tilde{K}=\tilde{L}$.

Lemma 3.4 Suppose that $(G, p)$ and $(G, q)$ are equivalent realizations of a minimally rigid mixed graph $G=(V ; D, L)$ and that $(G, p)$ is quasi-generic. Then $q(u) \neq q(v)$ for all $u v \in D$.

Proof: By applying suitable translations to $(G, p)$ and $(G, q)$ we may suppose that $p=\left(0,0, p_{3}, p_{4}, p_{5}, \ldots, p_{2 n}\right)$ and $q=\left(0,0, q_{3}, q_{4}, q_{5}, \ldots, q_{2 n}\right)$. Let $D_{0}=\{u v \in D: q(u)=q(v)\}$ and $H=G-D_{0}$. By Lemma 3.2, $f_{H}(p)$ is generic. Since $(G, p)$ and $(G, q)$ are equivalent, $(H, q)$ has no vertical direction edges. Hence $f_{H}(q)$ is well defined and we have $f_{H}(q)=f_{H}(p)$. Thus

$$
t d\left[\mathbb{Q}\left(f_{H}(q)\right): \mathbb{Q}\right]=\left|\left(D-D_{0}\right) \cup L\right|=2 n-2-\left|D_{0}\right| .
$$

Let $S=\{q(v): v \in V\}$. We have $t d[\mathbb{Q}(q): \mathbb{Q}] \leq 2|S|-2$ since $q(v)=(0,0)$ for at least one vertex $v \in V$. Since each coordinate of $f_{H}(q)$ is a ratio of two polynomial functions of the coordinates of $q$, we have $\mathbb{Q}\left(f_{H}(q)\right) \subseteq \mathbb{Q}(q)$. Thus

$$
t d\left[\mathbb{Q}\left(f_{H}(q)\right): \mathbb{Q}\right] \leq 2|S|-2 .
$$

Suppose that $D_{0} \neq \emptyset$. We shall show that, under this assumption, equations (1) and (2) give a contradiction. Let $V_{0}$ be the set of vertices of $G$ incident with edges of $D_{0}$. Let $F=\left(V_{0}, D_{0}\right)$, let $F_{1}, F_{2}, \ldots, F_{c}$ be the connected components of $F$, and put $V_{i}=V\left(F_{i}\right)$ for $1 \leq i \leq c$. Note that $F$ has minimum degree at least one so $\left|V_{i}\right| \geq 2$ for all $1 \leq i \leq c$. Since $G$ is independent and $F$ is a subgraph of $G, F$ is independent. Since $F$ is pure, we have $\left|E\left(F_{i}\right)\right| \leq 2\left|V_{i}\right|-3$ for all $1 \leq i \leq c$. Thus

$$
\left|D_{0}\right|=\sum_{i=1}^{c}\left|E\left(F_{i}\right)\right| \leq \sum_{i=1}^{c}\left(2\left|V_{i}\right|-3\right)=2 \sum_{i=1}^{c}\left|V_{i}\right|-3 c .
$$


The fact that, for each $i \in\{1,2, \ldots, c\}$, we have $q(u)=q(v)$ for all $u, v \in V_{i}$, implies that $|S| \leq n-\sum_{i=1}^{c}\left|V_{i}\right|+c$. Thus

$$
2|S|-2 \leq 2 n-2 \sum_{i=1}^{c}\left|V_{i}\right|+2 c-2 \leq 2 n-2-\left|D_{0}\right|-c .
$$

Substituting into (2), we contradict (1). Thus $D_{0}=\emptyset$.

Theorem 3.5 Suppose that $(G, p)$ is a rigid quasi-generic mixed framework, $(G, q)$ is equivalent to $(G, p)$, and $p(v)=(0,0)=q(v)$ for some $v \in V$. Then $(G, q)$ is quasi-generic and the algebraic closures of $\mathbb{Q}(p)$ and $\mathbb{Q}(q)$ are the same.

Proof: Let $H$ be a minimally rigid spanning subgraph of $G$. By Lemma $3.2, f_{H}(p)$ is generic. Lemma 3.4 and the facts that $(G, p)$ is quasi-generic and is equivalent to $(G, q)$ imply that $(G, q)$ has no vertical edges. Thus $f_{H}(q)$ is well defined and $f_{H}(q)=f_{H}(p)$ is generic. By Lemma 3.3, $(H, q)$ is quasi-generic and the algebraic closures of $\mathbb{Q}(p)$ and $\mathbb{Q}(q)$ are both equal to the algebraic closure of $\mathbb{Q}(f(p))$.

We close this section with an illustration of how the above results can be applied to a generic mixed framework which is 'almost' rigid.

Lemma 3.6 Let $G=(V ; D, L)$ be a mixed graph with $r(G)=2|V|-3$, and let $(G, p)$ and $(G, q)$ be equivalent realizations of $G$. Suppose that $(G, p)$ is quasi-generic and that the set $U=\{u \in V: q(u)=q(v)$ for some $v \in$ $V-u\}$ is not empty. Then $U$ induces a maximal direction rigid subgraph of $G$ and $q(u)=q(v)$ for all $u, v \in U$.

Proof: We first consider the case when $G$ is independent, and hence $|D|+$ $|L|=2|V|-3$. The proof of Lemma 3.4 applies to $G$ word by word until the end of (4), except that (1) must be replaced by

$$
t d\left[\mathbb{Q}\left(f_{H}(q)\right): \mathbb{Q}\right]=\left|\left(D-D_{0}\right) \cup L\right|=2 n-3-\left|D_{0}\right|,
$$

due to the fact that $G$ now has one less edge. We can use (5), (2), (3), and (4) to deduce that $c=1$ and hence $F_{1}=F=\left(V_{0}, D_{0}\right)$. Furthermore, equality must hold in (3) and (4), so $\left|D_{0}\right|=2\left|V_{0}\right|-3$ and $|S|=n-\left|V_{0}\right|+1$. This implies that $U=V_{0}$ and, by using the fact that $G$ is independent, that $\left(V_{0}, D_{0}\right)$ is direction rigid. This in turn implies that $U$ induces a maximal direction rigid subgraph of $G$ and $q(u)=q(v)$ for all $u, v \in U$. 
When $G$ is not independent we may apply the above argument to a maximal independent subgraph $H$ of $G$ to deduce that $U$ induces a maximal direction rigid subgraph of $H$ and $q(u)=q(v)$ for all $u, v \in U$. The fact that $r(G)=r(H)$ now implies that $U$ also induces a maximal direction rigid subgraph of $G$.

Lemma 3.6 has implications for the continuous motion of a generic mixed framework $(G, p)$ with $r(G)=2|V|-3$. It is possible that such a motion may arrive at an equivalent framework $(G, q)$ with $q(u)=q(v)$ for distinct $u, v \in V$. (Consider for example the graph consisting of two vertices joined by one direction edge.) Lemma 3.6 tells us that if this does occur then all co-incindent pairs of vertices of $(G, q)$ are mapped onto the same point, and that the union of all these pairs induces a maximal direction rigid subgraph of $G$. A special case of this result when $|L|=1$ is stated by Streinu in [15, Theorem 4].

\section{Extensions and globally linked pairs}

In this section we prove that mixed versions of the Henneberg operations preserve global rigidity. We first prove this for the 0-extension operation.

Theorem 4.1 Let $G=(V ; D, L)$ be a mixed graph, $v \in V$, with $d(v)=2$ and $v u, v w \in D$, and $H=G-v$. Then $G$ is globally rigid if and only if $H$ is globally rigid.

Proof: We first suppose that $H$ is globally rigid. Let $(G, p)$ be a generic framework and $(G, q)$ be equivalent to $(G, p)$. Since $H$ is globally rigid, we may assume (by applying a suitable translation and/or dilation by -1 ) that $\left.p\right|_{H}=\left.q\right|_{H}$. In particular, $p(u)=q(u)$ and $p(w)=q(w)$. Since $v u, v w \in D$, this implies that $p(v)=q(v)$. Thus $(G, p)$ and $(G, q)$ are congruent. Hence $G$ is globally rigid.

We next suppose that $G$ is globally rigid. Let $\left(H, p^{\prime}\right)$ be a generic framework and $\left(H, q^{\prime}\right)$ be equivalent to $\left(H, p^{\prime}\right)$. Choose a point $P \in \mathbb{R}^{2}$ such that $p^{\prime}(V-v) \cup\{P\}$ is generic. Let $(G, p)$ be the generic realization of $G$ with $p(x)=p^{\prime}(x)$ for all $x \in V-v$ and $p(v)=P$. Let $Q$ be the point of intersection of the lines through $q^{\prime}(u)$ and $q^{\prime}(w)$ with slopes $s_{p}(v, u)$ and $s_{p}(v, w)$, respectively. Let $(G, q)$ be the realization of $G$ with $q(x)=q^{\prime}(x)$ for all $x \in V-v$ and $q(v)=Q$. Then $(G, p)$ and $(G, q)$ are equivalent. Since $G$ is globally rigid, $(G, p)$ and $(G, q)$ are congruent. Hence $\left(H, p^{\prime}\right)$ and $\left(H, q^{\prime}\right)$ 
are congruent.

To prove a similar result concerning 1-extensions we need a few more definitions. Let $(G, p)$ be a generic mixed framework and $u, v \in V$. The pair $\{u, v\}$ is globally distance linked, respectively globally direction linked, in $(G, p)$ if, in all equivalent frameworks $(G, q)$, we have $l_{p}(u, v)=l_{q}(u, v)$, respectively $s_{p}(u, v)=s_{q}(u, v)$. It is globally linked in $(G, p)$ if it is both globally distance linked and globally direction linked. The pair $\{u, v\}$ is globally distance linked, respectively globally direction linked or globally linked, in $G$ if it is globally distance linked, respectively globally direction linked or globally linked, in all generic frameworks $(G, p)$. These notions were introduced for length frameworks in [9]. Note that a mixed graph $G$ is globally rigid if and only if all pairs of vertices of $G$ are globally linked. As an example consider the mixed graph in Figure 5. The results of the next section will imply that the vertex pair of the 2-separator of this mixed graph is globally linked even though the whole graph is not globally rigid.

Consider a 1-extension operation in a mixed graph $H$ which deletes the edge $u w$ and adds a new vertex $v$ joined to vertices $u, w, t$. The next lemma will imply that the pairs of neighbours of $v$ in the extended graph $G$ are globally (direction and/or distance) linked, depending on the types of edges incident with $v$, provided $G-v=H-u w$ is rigid. As an easy corollary, we shall then deduce that $G$ is globally rigid if $H$ is globally rigid and $H-u w$ is rigid.

The proof of this lemma is somewhat lengthy, due to the fact that we have to perform extensive (but elementary) calculations on systems of polynomial equations and we need to consider three cases, depending on whether the edges incident with $v$ are all direction edges, all length edges, or are mixed. Before presenting the details we sketch the main ideas of the proof. In each of the three cases we consider a quasi-generic realization $(G, p)$ of $G$ and an equivalent realization $(G, q)$. Let $\left\{v_{1}, v_{2}, \ldots, v_{n}\right\}$ be the set of vertices of $G$, where $v_{1}=u, v_{2}=w, v_{3}=t$, and $v_{n}=v$. We may suppose without loss of generality that $p\left(v_{1}\right)=(0,0), p\left(v_{i}\right)=\left(p_{2 i-1}, p_{2 i}\right)$ for $2 \leq i \leq n$, $q\left(v_{1}\right)=(0,0)$, and $q\left(v_{i}\right)=\left(q_{2 i-1}, q_{2 i}\right)$ for $2 \leq i \leq n$. By Lemma 3.1, $\left\{p_{3}, p_{4}, \ldots, p_{2 n}\right\}$ is algebraically independent over $\mathbb{Q}$.

Let $p^{\prime}=\left.p\right|_{V-v}$ and $q^{\prime}=\left.q\right|_{V-v}$. Consider the equivalent frameworks $\left(G-v, p^{\prime}\right)$ and $\left(G-v, q^{\prime}\right)$. Since $\left\{p_{3}, p_{4}, \ldots, p_{2 n}\right\}$ is generic, $\left(G-v, p^{\prime}\right)$ is quasi-generic. Since $G-v$ is rigid, we may apply Theorem 3.5 to $G-v$ to deduce that $\tilde{K}=\tilde{L}$ where $K=\mathbb{Q}\left(p^{\prime}\right)$ and $L=\mathbb{Q}\left(q^{\prime}\right)$. Thus $q_{3}, q_{4}, q_{5}, q_{6} \in \tilde{K}$ and $q_{3}, q_{4}, q_{5}, q_{6}$ are algebraically independent over $\mathbb{Q}$. 
The three edges (constraints) incident with $v$ and the equivalence of $(G, p)$ and $(G, q)$ give rise to three polynomial equations for the coordinates of $v$ and its three neighbours in the two realizations of $G$. From these equations we may obtain a polynomial $f \in \tilde{K}\left[z_{1}, z_{2}\right]$ for which $f\left(p_{2 n-1}, p_{2 n}\right)=0$, and whose coefficients are polynomial functions of the coordinates of the neighbours of $v$. Since $\left\{p_{3}, p_{4}, \ldots, p_{2 n}\right\}$ is algebraically independent over $\mathbb{Q}$, $\left\{p_{2 n-1}, p_{2 n}\right\}$ is algebraically independent over $\tilde{K}$. Thus $f \equiv 0$, which implies that each coefficient is zero. This gives rise to further polynomial equations which can be used to deduce that the pairs of neighbours of $v$ must be linked. For example, by deducing that $p_{4} / p_{3}=q_{4} / q_{3}$ we may conclude that the pair $(u, w)$ is globally direction linked.

Lemma 4.2 Let $G=(V ; D, L)$ be a mixed graph and $v \in V(G)$ with $d(v)=$ 3. Let $v u, v w, v t$ be the edges incident to $v$ and suppose that $G-v$ is rigid. (a) If $\{v u, v w, v t\} \subseteq D$, then $\{u, w\},\{u, t\}$ and $\{w, t\}$ are globally direction linked in $G$.

(b) If $\{v u, v w, v t\} \subseteq L$, then $\{u, w\},\{u, t\}$ and $\{w, t\}$ are globally distance linked in $G$.

(c) If $\{v u, v w, v t\} \cap D \neq \emptyset \neq\{v u, v w, v t\} \cap L$, then $\{u, w\},\{u, t\}$ and $\{w, t\}$ are globally linked in $G$.

Proof: Note that $v$ must have three distinct neighbours in cases (a) and (b), but may only have two distinct neighbours in case (c). Relabelling if necessary, we may suppose that $u \neq w \neq t$ in all cases. Let $V=\left\{v_{1}, v_{2}, \ldots, v_{n}\right\}$, where $v_{1}=u, v_{2}=w, v_{3}=t$ if $u \neq t$, and $v_{n}=v$. Let $(G, p)$ be a quasigeneric realization of $G$ and $(G, q)$ be an equivalent realization. We may suppose without loss of generality that $p\left(v_{1}\right)=(0,0), p\left(v_{i}\right)=\left(p_{2 i-1}, p_{2 i}\right)$ for $2 \leq i \leq n, q\left(v_{1}\right)=(0,0)$, and $q\left(v_{i}\right)=\left(q_{2 i-1}, q_{2 i}\right)$ for $2 \leq i \leq n$. By Lemma $3.1,\left\{p_{3}, p_{4}, \ldots, p_{2 n}\right\}$ is algebraically independent over $\mathbb{Q}$.

Let $p^{\prime}=\left.p\right|_{V-v}$ and $q^{\prime}=\left.q\right|_{V-v}$. Consider the equivalent frameworks $\left(G-v, p^{\prime}\right)$ and $\left(G-v, q^{\prime}\right)$. Since $\left\{p_{3}, p_{4}, \ldots, p_{2 n}\right\}$ is generic, $\left(G-v, p^{\prime}\right)$ is quasi-generic. Since $G-v$ is rigid, we may apply Theorem 3.5 to $G-v$ to deduce that $\tilde{K}=\tilde{L}$ where $K=\mathbb{Q}\left(p^{\prime}\right)$ and $L=\mathbb{Q}\left(q^{\prime}\right)$. Thus $q_{3}, q_{4}, q_{5}, q_{6} \in \tilde{K}$ and $q_{3}, q_{4}, q_{5}, q_{6}$ are algebraically independent over $\mathbb{Q}$.

Proof of (a) Since $(G, q)$ is equivalent to $(G, p)$, we have the following equations:

$$
\begin{aligned}
q_{2 n} / q_{2 n-1} & =p_{2 n} / p_{2 n-1} \\
\left(q_{2 n}-q_{4}\right) /\left(q_{2 n-1}-q_{3}\right) & =\left(p_{2 n}-p_{4}\right) /\left(p_{2 n-1}-p_{3}\right) \\
\left(q_{2 n}-q_{6}\right) /\left(q_{2 n-1}-q_{5}\right) & =\left(p_{2 n}-p_{6}\right) /\left(p_{2 n-1}-p_{5}\right) .
\end{aligned}
$$


We may rewrite each of the above equations as:

$$
\begin{aligned}
q_{2 n} p_{2 n-1} & =p_{2 n} q_{2 n-1} \\
q_{2 n}\left(p_{2 n-1}-p_{3}\right)-q_{2 n-1}\left(p_{2 n}-p_{4}\right) & =q_{4}\left(p_{2 n-1}-p_{3}\right)-q_{3}\left(p_{2 n}-p_{4}\right)(10) \\
q_{2 n}\left(p_{2 n-1}-p_{5}\right)-q_{2 n-1}\left(p_{2 n}-p_{6}\right) & =q_{6}\left(p_{2 n-1}-p_{5}\right)-q_{5}\left(p_{2 n}-p_{6}\right)(11)
\end{aligned}
$$

Using equations (9) and (10) we obtain

$$
q_{2 n-1} p_{4}-q_{2 n} p_{3}=q_{3}\left(p_{4}-p_{2 n}\right)-q_{4}\left(p_{3}-p_{2 n-1}\right) .
$$

Similarly, using equations (9) and (11), we obtain

$$
q_{2 n-1} p_{6}-q_{2 n} p_{5}=q_{5}\left(p_{6}-p_{2 n}\right)-q_{6}\left(p_{5}-p_{2 n-1}\right) .
$$

We may solve (12) and (13) for $q_{2 n-1}$ and $q_{2 n}$ and then substitute into (9) to obtain

$$
a_{2,0} p_{2 n-1}^{2}+a_{0,2} p_{2 n}^{2}+a_{1,1} p_{2 n-1} p_{2 n}+a_{1,0} p_{2 n-1}+a_{0,1} p_{2 n}+a_{0,0}=0,
$$

where $a_{i, j} \in \tilde{K}$. This means, that there is a polynomial

$$
f=a_{2,0} z_{1}^{2}+a_{0,2} z_{2}^{2}+a_{1,1} z_{1} z_{2}+a_{1,0} z_{1}+a_{0,1} z_{2}+a_{0,0} \in \tilde{K}\left[z_{1}, z_{2}\right]
$$

such that $f\left(p_{2 n-1}, p_{2 n}\right)=0$. Since $\left\{p_{3}, p_{4}, \ldots, p_{2 n}\right\}$ is algebraically independent over $\mathbb{Q},\left\{p_{2 n-1}, p_{2 n}\right\}$ is algebraically independent over $\tilde{K}$. Thus $f \equiv 0$. We have, $a_{2,0}=q_{4} p_{6}-q_{6} p_{4}, a_{0,2}=q_{3} p_{5}-q_{5} p_{3}$ and $a_{1,1}=$ $q_{6} p_{3}-q_{4} p_{5}+q_{5} p_{4}-q_{3} p_{6}$. Putting $a_{2,0}=0$ we obtain $p_{6}=q_{6} p_{4} / q_{4}$. Similarly, $a_{0,2}=0$ implies that $p_{5}=q_{5} p_{3} / q_{3}$. We may substitute these values for $p_{5}, p_{6}$ into the equation $a_{1,1}=0$ to obtain $p_{4} / p_{3}=q_{4} / q_{3}$. Thus the pair $(u, w)$ is globally direction linked. Symmetry now implies that $(u, w)$ and $(w, t)$ are also globally direction linked.

Proof of (b) Since $(G, q)$ is equivalent to $(G, p)$, we have the following equations:

$$
\begin{aligned}
q_{2 n-1}^{2}+q_{2 n}^{2} & =p_{2 n-1}^{2}+p_{2 n}^{2} \\
\left(q_{2 n-1}-q_{3}\right)^{2}+\left(q_{2 n}-q_{4}\right)^{2} & =\left(p_{2 n-1}-p_{3}\right)^{2}+\left(p_{2 n}-p_{4}\right)^{2} \\
\left(q_{2 n-1}-q_{5}\right)^{2}+\left(q_{2 n}-q_{6}\right)^{2} & =\left(p_{2 n-1}-p_{5}\right)^{2}+\left(p_{2 n}-p_{6}\right)^{2}
\end{aligned}
$$

Using equations (14) and (15) we obtain

$$
q_{3}\left(2 q_{2 n-1}-q_{3}\right)+q_{4}\left(2 q_{2 n}-q_{4}\right)=p_{3}\left(2 p_{2 n-1}-p_{3}\right)+p_{4}\left(2 p_{2 n}-p_{4}\right) .
$$


Similarly, using equations (14) and (16), we obtain

$$
q_{5}\left(2 q_{2 n-1}-q_{5}\right)+q_{6}\left(2 q_{2 n}-q_{6}\right)=p_{5}\left(2 p_{2 n-1}-p_{5}\right)+p_{6}\left(2 p_{2 n}-p_{6}\right) .
$$

We may solve (17) and (18) for $q_{2 n-1}$ and $q_{2 n}$ and then substitute into (14) to obtain

$$
a_{2,0} p_{2 n-1}^{2}+a_{0,2} p_{2 n}^{2}+a_{1,1} p_{2 n-1} p_{2 n}+a_{1,0} p_{2 n-1}+a_{0,1} p_{2 n}+a_{0,0}=0,
$$

where $a_{i, j} \in \tilde{K}$. We may deduce, as in (a), that $a_{i, j}=0$ for all $0 \leq i+j \leq 2$. In particular,

$$
\begin{aligned}
a_{2,0}= & \left(p_{3} q_{6}-p_{5} q_{4}\right)^{2}+\left(p_{3} q_{5}-p_{5} q_{3}\right)^{2}-\left(q_{3} q_{6}-q_{5} q_{4}\right)^{2}=0 \\
a_{0,2}= & \left(p_{4} q_{6}-p_{6} q_{4}\right)^{2}+\left(p_{4} q_{5}-p_{6} q_{3}\right)^{2}-\left(q_{3} q_{6}-q_{5} q_{4}\right)^{2}=0 \\
a_{1,1}= & p_{3}\left(p_{4} q_{6}^{2}+p_{4} q_{5}^{2}-p_{6} q_{3} q_{5}-p_{6} q_{4} q_{6}\right)+ \\
& p_{5} p_{6} q_{3}^{2}+p_{5} p_{6} q_{4}^{2}-p_{4} p_{5} q_{4} q_{5}-p_{4} p_{5} q_{3} q_{5}=0 .
\end{aligned}
$$

We may solve the $a_{1,1}$-equation for $p_{3}$ and substitute into the $a_{2,0}$-equation to obtain $\left(q_{3} q_{6}-q_{5} q_{4}\right)^{2} b_{2,0}=0$, where

$b_{2,0}=p_{5}^{2}\left[\left(p_{4} q_{6}-p_{6} q_{4}\right)^{2}+\left(p_{4} q_{5}-p_{6} q_{3}\right)^{2}\right]-\left[q_{6}\left(p_{4} q_{6}-p_{6} q_{4}\right)+q_{5}\left(p_{4} q_{5}-p_{6} q_{3}\right)\right]^{2}$.

Since $q_{3}, q_{4}, q_{5}, q_{6}$ are algebraically independent over $\mathbb{Q}$, we have $\left(q_{3} q_{6}-\right.$ $\left.q_{5} q_{4}\right)^{2} \neq 0$ and hence $b_{2,0}=0$. If we now use the $a_{0,2}$-equation to replace $p_{5}^{2}\left(p_{4} q_{6}-p_{6} q_{4}\right)^{2}+\left(p_{4} q_{5}-p_{6} q_{3}\right)^{2}, q_{6}^{2}\left(p_{4} q_{6}-p_{6} q_{4}\right)^{2}$, and $q_{5}^{2}\left(p_{4} q_{5}-p_{6} q_{3}\right)^{2}$ by $p_{5}^{2}\left(q_{3} q_{6}-q_{5} q_{4}\right)^{2}, q_{6}^{2}\left[\left(q_{3} q_{6}-q_{5} q_{4}\right)^{2}-\left(p_{4} q_{5}-p_{6} q_{3}\right)^{2}\right]$, and $q_{5}^{2}\left[\left(q_{3} q_{6}-q_{5} q_{4}\right)^{2}-\right.$ $\left.\left(p_{4} q_{6}-p_{6} q_{4}\right)^{2}\right]$, respectively, in the $b_{2,0}$-equation, we obtain

$$
\left(q_{3} q_{6}-q_{5} q_{4}\right)^{2}\left(p_{5}^{2}+p_{6}^{2}-q_{5}^{2}-q_{6}^{2}\right)=0 .
$$

Since $\left(q_{3} q_{6}-q_{5} q_{4}\right)^{2} \neq 0$, this gives $\left(p_{5}^{2}+p_{6}^{2}\right)-\left(q_{5}^{2}+q_{6}^{2}\right)=0$. Thus the pair $(u, t)$ is globally distance linked. Symmetry now implies that $(u, w)$ and $(w, t)$ are also globally distance linked.

Proof of (c) We need to consider two cases, depending on whether $v$ is incident to two direction edges or two length edges.

Case 1: $v u \in L$ and $v w, v t \in D$.

We first consider the subcase when $u \neq t$. Since $(G, q)$ is equivalent to $(G, p)$, we have the following equations:

$$
\begin{aligned}
q_{2 n-1}^{2}+q_{2 n}^{2} & =p_{2 n-1}^{2}+p_{2 n}^{2} \\
\left(q_{2 n}-q_{4}\right) /\left(q_{2 n-1}-q_{3}\right) & =\left(p_{2 n}-p_{4}\right) /\left(p_{2 n-1}-p_{3}\right) \\
\left(q_{2 n}-q_{6}\right) /\left(q_{2 n-1}-q_{5}\right) & =\left(p_{2 n}-p_{6}\right) /\left(p_{2 n-1}-p_{5}\right) .
\end{aligned}
$$


Using equation (20) we obtain

$$
q_{2 n-1}\left(p_{4}-p_{2 n}\right)-q_{2 n}\left(p_{3}-p_{2 n-1}\right)=q_{3}\left(p_{4}-p_{2 n}\right)-q_{4}\left(p_{3}-p_{2 n-1}\right) .
$$

Similarly, using equation (21), we obtain

$$
q_{2 n-1}\left(p_{6}-p_{2 n}\right)-q_{2 n}\left(p_{5}-p_{2 n-1}\right)=q_{5}\left(p_{6}-p_{2 n}\right)-q_{6}\left(p_{5}-p_{2 n-1}\right) .
$$

We may solve (22) and (23) for $q_{2 n-1}$ and $q_{2 n}$ and then substitute into (19) to obtain $\sum_{0 \leq i+j \leq 4} a_{i, j} p_{2 n-1}^{i} p_{2 n}^{j}=0$, where $a_{i, j} \in \tilde{K}$ for all $0 \leq i+j \leq 6$. Again we have $a_{i, j}=0$ for all $0 \leq i+j \leq 4$. In particular,

$$
\begin{aligned}
a_{4,0}= & \left(p_{6}-p_{4}\right)^{2}-\left(q_{6}-q_{4}\right)^{2}=0 \\
a_{3,1}= & 2\left(\left(p_{5}-p_{3}\right)\left(p_{6}-p_{4}\right)-\left(q_{5}-q_{3}\right)\left(q_{6}-q_{4}\right)\right)=0 \\
a_{3,0}= & 2\left[\left(p_{6}-p_{4}\right)\left(p_{4} p_{5}-p_{6} p_{3}\right)+\left(q_{6}-q_{4}\right)\left(p_{4} q_{3}-p_{6} q_{5}\right)+\right. \\
& \left.\left(q_{6}-q_{4}\right)^{2}\left(p_{5}+p_{3}\right)\right]=0 \\
a_{0,3}= & 2\left[\left(p_{5}-p_{3}\right)\left(p_{3} p_{6}-p_{5} p_{4}\right)+\left(q_{5}-q_{3}\right)\left(p_{3} q_{4}-p_{5} q_{6}\right)+\right. \\
& \left.\left(q_{5}-q_{3}\right)^{2}\left(p_{6}+p_{4}\right)\right]=0 .
\end{aligned}
$$

The $a_{4,0}$-equation tells us that $q_{6}-q_{4}=\alpha\left(p_{6}-p_{4}\right)$ for some $\alpha \in\{1,-1\}$. Substituting into the $a_{3,1^{-}}$and $a_{3,0}$-equations, we obtain $q_{5}-q_{3}=\alpha\left(p_{5}-p_{3}\right)$, and $\left(p_{6}-p_{4}\right)\left(p_{6}\left[p_{5}-\alpha q_{5}\right]-p_{4}\left[p_{3}-\alpha q_{3}\right]\right)=0$. Since $p_{6} \neq p_{4}$, we may use both equations to deduce that $p_{3}=\alpha q_{3}$ and $p_{5}=\alpha q_{5}$. A similar argument using the $a_{4,0^{-}}, a_{3,1^{-}}$and $a_{0,3^{-}}$equations gives $p_{4}=\alpha q_{4}$ and $p_{6}=\alpha q_{6}$. Hence either $p_{i}=q_{i}$ for all $3 \leq i \leq 6$ or $p_{i}=-q_{i}$ for all $3 \leq i \leq 6$. Thus $\{u, w\}$, $\{u, t\}$ and $\{w, t\}$ are globally linked in $G$.

The subcase when $u=t$ can be handled similarly. We replace each of $p_{5}, p_{6}, q_{5}, q_{6}$ by zero in the above analysis. The resulting $a_{4,0^{-}}$and $a_{3,1^{-}}$ equations then imply that $\left(p_{3}, p_{4}\right)= \pm\left(q_{3}, q_{4}\right)$ and hence $(u, w)$ is globally linked in $G$.

Case 2: $v u \in D$ and $v w, v t \in L$.

We first consider the subcase when $u \neq t$. Since $(G, q)$ is equivalent to $(G, p)$, we have the following equations:

$$
\begin{aligned}
q_{2 n} / q_{2 n-1} & =p_{2 n} / p_{2 n-1} \\
\left(q_{2 n-1}-q_{3}\right)^{2}+\left(q_{2 n}-q_{4}\right)^{2} & =\left(p_{2 n-1}-p_{3}\right)^{2}+\left(p_{2 n}-p_{4}\right)^{2} \\
\left(q_{2 n-1}-q_{5}\right)^{2}+\left(q_{2 n}-q_{6}\right)^{2} & =\left(p_{2 n-1}-p_{5}\right)^{2}+\left(p_{2 n}-p_{6}\right)^{2} .
\end{aligned}
$$


Using equations (25) and (26) we obtain

$$
\begin{aligned}
& \left(2 q_{2 n-1}-q_{3}-q_{5}\right)\left(q_{5}-q_{3}\right)+\left(2 q_{2 n}-q_{4}-q_{6}\right)\left(q_{6}-q_{4}\right)= \\
& \quad\left(2 p_{2 n-1}-p_{3}-p_{5}\right)\left(p_{5}-p_{3}\right)+\left(2 p_{2 n}-p_{4}-p_{6}\right)\left(p_{6}-p_{4}\right) .
\end{aligned}
$$

We may solve (24) and (27) for $q_{2 n-1}$ and $q_{2 n}$ and then substitute into (25) to obtain $\sum_{0 \leq i+j \leq 4} a_{i, j} p_{2 n-1}^{i} p_{2 n}^{j}=0$, where $a_{i j} \in \tilde{K}$ for all $0 \leq i+j \leq 4$. Again we have $a_{i, j}=0$ for all $0 \leq i+j \leq 4$. In particular,

$$
\begin{aligned}
& a_{4,0}=\left(q_{5}-q_{3}\right)^{2}-\left(p_{5}-p_{3}\right)^{2}=0 \\
& a_{0,4}=\left(q_{6}-q_{4}\right)^{2}-\left(p_{6}-p_{4}\right)^{2}=0 \\
& a_{3,1}=2\left(-p_{5} p_{6}-p_{3} p_{4}+p_{3} p_{6}+p_{4} p_{5}+q_{5} q_{6}-q_{3} q_{6}-q_{5} q_{4}+q_{3} q_{4}\right)=0 \\
& a_{3,0}=\left(p_{5}-p_{3}\right)\left(p_{5}^{2}-p_{3}^{2}+p_{6}^{2}-p_{4}^{2}+q_{4}^{2}-q_{6}^{2}\right)-\left(p_{5}+p_{3}\right)\left(q_{5}-q_{3}\right)^{2}=0 \\
& a_{0,3}=\left(p_{6}-p_{4}\right)\left(p_{6}^{2}-p_{4}^{2}+p_{5}^{2}-p_{3}^{2}+q_{3}^{2}-q_{5}^{2}\right)-\left(p_{6}+p_{4}\right)\left(q_{6}-q_{4}\right)^{2}=0
\end{aligned}
$$

The $a_{4,0}$-equation tells us that $\left(q_{5}-q_{3}\right)^{2}=\left(p_{5}-p_{3}\right)^{2}$. We may use this to replace $\left(q_{5}-q_{3}\right)^{2}$ by $\left(p_{5}-p_{3}\right)^{2}$ in the last term of the $a_{3,0}$ equation to obtain $\left(p_{5}-p_{3}\right)\left(p_{6}^{2}-p_{4}^{2}+q_{4}^{2}-q_{6}^{2}\right)=0$. Since $\left\{p_{3}, p_{4}, \ldots, p_{2 n}\right\}$ is algebraically independent over $\mathbb{Q},\left(p_{5}-p_{3}\right) \neq 0$. Thus $p_{6}^{2}-p_{4}^{2}=q_{6}^{2}-q_{4}^{2}$. We may combine this with the $a_{4,0}$-equation to deduce that $p_{4}^{2}=q_{4}^{2}$ and $p_{6}^{2}=q_{6}^{2}$. A similar analysis using the $a_{0,4^{-}}$and $a_{0,3^{-}}$-equations yeilds $p_{3}^{2}=q_{3}^{2}$ and $p_{5}^{2}=q_{5}^{2}$. Thus $p_{i}=\alpha_{i} q_{i}$ for some $\alpha_{i} \in\{1,-1\}$ and all $3 \leq i \leq 6$. Substituting into the $a_{3,1}$-equation we obtain

$$
\left(1-\alpha_{5} \alpha_{6}\right) q_{5} q_{6}+\left(1-\alpha_{3} \alpha_{4}\right) q_{3} q_{4}+\left(\alpha_{3} \alpha_{6}-1\right) q_{3} q_{6}+\left(\alpha_{4} \alpha_{5}-1\right) q_{4} q_{5}=0 .
$$

Since $\left\{q_{3}, q_{4}, q_{5}, q_{6}\right\}$ is algebraically independent over $\mathbb{Q}$, all coefficients must be zero. This implies that all the $\alpha_{i}$ are equal and hence either $p_{i}=q_{i}$ for all $3 \leq i \leq 6$ or $p_{i}=-q_{i}$ for all $3 \leq i \leq 6$. Thus $\{u, w\},\{u, t\}$ and $\{w, t\}$ are globally linked in $G$.

The subcase when $u=t$ can be handled similarly. We replace each of $p_{5}, p_{6}, q_{5}, q_{6}$ by zero in the above analysis. The resulting $a_{4,0^{-}}, a_{0,4^{-}}$and $a_{3,1^{-}}$ equations then imply that $\left(p_{3}, p_{4}\right)= \pm\left(q_{3}, q_{4}\right)$ and hence $(u, w)$ is globally linked in $G$.

Theorem 4.3 Let $G$ be a 1-extension of a mixed graph $H$, so $H=G-$ $v+u w$ for some vertex $v$ of $G$ where $u, w$ are neighbours of $v$. Suppose that 
$H-u w$ is rigid and that $x, y$ are vertices of $H$. If $(x, y)$ is globally linked in $H$ then $(x, y)$ is globally linked in $G$.

Proof: Suppose $(G, p)$ is a generic mixed framework and that $(G, q)$ is equivalent to $(G, p)$. Let $p^{\prime}=\left.p\right|_{V-v}$ and $q^{\prime}=\left.q\right|_{V-v}$. Since $G-v=H-u w$ is rigid, Lemma 4.2 implies that $\{u, w\}$ is globally direction linked in $G$ if $G$ is a direction 1-extension of $H$, that $\{u, w\}$ is globally distance linked in $G$ if $G$ is a distance 1-extension of $H$, and that $\{u, w\}$ is globally linked in $G$ if $G$ is a mixed 1-extension of $H$. Thus $\left(H, p^{\prime}\right)$ and $\left(H, q^{\prime}\right)$ are equivalent. Since $\{x, y\}$ is globally linked in $H$, we have

$$
l_{p}(x, y)=l_{p^{\prime}}(x, y)=l_{q^{\prime}}(x, y)=l_{q}(x, y)
$$

and

$$
s_{p}(x, y)=s_{p^{\prime}}(x, y)=s_{q^{\prime}}(x, y)=s_{q}(x, y) .
$$

Thus $\{x, y\}$ is globally linked in $G$.

We can now deduce our second result on mixed versions of the Henneberg operations.

Theorem 4.4 Let $H$ be a globally rigid mixed graph with $|V(H)| \geq 3$ and $G$ be obtained from $H$ by a 1-extension, which deletes an edge uw and adds a vertex $v$ joined to vertices $u, w, t$. Suppose that $H-u w$ is rigid. Then $G$ is globally rigid.

Proof: Theorem 4.3 and the fact that $H$ is globally rigid imply that all pairs $\{x, y\} \subseteq V-v$ are globally linked in $G$. Suppose $(G, p)$ is a generic framework and that $(G, q)$ is equivalent to $(G, p)$. Let $p^{\prime}=\left.p\right|_{V-v}$ and $q^{\prime}=\left.q\right|_{V-v}$. Since all pairs $\{x, y\} \subseteq V-v$ are globally linked in $G$, we may assume (by applying a suitable translation and/or rotation by $180^{\circ}$ to $(G, p)$ ) that $p^{\prime}=q^{\prime}$. Since $(G, p)$ is generic and $d_{G}(v)=3$, this implies that we must also have $p(v)=q(v)$. Thus $(G, p)$ and $(G, q)$ are congruent.

Note that Theorems 1.3 and 1.4 are slightly reformulated versions of Theorems 4.1 and 4.4, respectively.

\section{Globally linked pairs in mixed circuits}

The notion of global linkedness, which was introduced and used in the previous section to prove our main results on 1-extensions, may also be useful 
in applications (for example in the localization problem of sensor networks) and leads to the general question of how to identify globally linked pairs in mixed graphs.

The results of Section 4, together with some results from [10], can be used to characterize the globally linked pairs and the 'globally rigid clusters' in mixed circuits. We shall need the following three lemmas. A mixed graph $G$ is a pure circuit if the edge set of $G$ is pure and forms a circuit in the direction-length rigidity matroid of $G$.

Lemma 5.1 [10, Lemma 3.7] Let $G$ be a mixed graph.

(a) Suppose $G$ is the 2-sum of two mixed graphs $G_{1}$ and $G_{2}$. If $G_{1}$ is a mixed circuit and $G_{2}$ is a pure circuit, then $G$ is a mixed circuit.

(b) Suppose $G$ is a mixed circuit and $\left(H_{1}, H_{2}\right)$ is a 2-separation of $G$, where $V\left(H_{1}\right) \cap V\left(H_{2}\right)=\{u, v\}$ and $H_{2}$ is pure. Let $G_{i}$ be obtained from $H_{i}$ by adding a new edge uv of the same type as the edges of $H_{2}$. Then $G_{1}$ is a mixed circuit, $G_{2}$ is a pure circuit and $G=G_{1} \oplus_{2} G_{2}$.

Lemma 5.2 Let $G_{1}$ be a mixed circuit and $G_{2}$ be a 3-connected pure length circuit. Let $G=G_{1} \oplus_{2} G_{2}$, where the 2-sum is obtained along the length edge $x y \in E\left(G_{1}\right) \cap E\left(G_{2}\right)$. Then $\{x, y\}$ is globally distance linked in $G$.

Proof: We will use induction on $\left|V\left(G_{2}\right)\right|$. If $G_{2}=K_{4}$, with $V\left(G_{2}\right)=$ $\{v, t, x, y\}$, then $G-v=G_{1}-x y+t+\{t x, t y\}$. Since $G_{1}$ is redundantly rigid, $G_{1}-x y$, and hence also $G-v$, are rigid. By Lemma 4.2(b), $\{x, y\}$ is globally distance linked in $G$. Thus we may suppose that $\left|V\left(G_{2}\right)\right| \geq 5$.

By [2, Theorem 5.9] there exists a vertex $v \in V\left(G_{2}\right)-\{x, y\}$, with $N(v)=\{u, w, t\}$, such that $G_{2}^{v}=G_{2}-v+u w$ is a 3-connected pure length circuit. Let $H=G_{1} \oplus_{2} G_{2}^{v}$ be the 2-sum along the edge $x y$. By induction, $\{x, y\}$ is globally distance-linked in $H$. Since $H$ is a mixed circuit by Lemma 5.1(a), $H-u w$ is rigid. Lemma $4.2(\mathrm{~b})$ now implies that $\{u, w\}$ is globally distance linked in $G$. Since $\{x, y\}$ is globally distance-linked in $H$ and $H-u w \subseteq G$, it follows that $\{x, y\}$ is globally distance-linked in $G$.

Lemma 5.3 Let $G$ be a mixed circuit and $\{u, v\}$ be a direction unbalanced 2-separator of $G$. Then $\{u, v\}$ is globally distance linked in $G$.

Proof: We will use induction on $|V(G)|$. Since $G$ has a direction unbalanced 2-separator, it follows from Lemma 5.1(b) that we can express $G$ as $G=$ $G_{1} \oplus_{2} G_{2}$, where $G_{1}$ is a mixed circuit and $G_{2}$ is a pure length circuit. Suppose that the 2-sum is obtained along the length edge $x y \in E\left(G_{1}\right) \cap E\left(G_{2}\right)$. 
Then $x y$ is not a length edge of $G$. By choosing $\{x, y\}$ so that $G_{2}$ is minimal, we may also ensure that $G_{2}$ is 3 -connected. By Lemma $5.2,\{x, y\}$ is globally distance linked in $G$. Thus we may suppose that $\{u, v\} \neq\{x, y\}$. Since $G_{2}$ is 3 -connected, $\{u, v\}$ is a 2 -separator of $G_{1}$. By induction, $\{u, v\}$ is globally distance linked in $G_{1}$. Since $\{x, y\}$ is globally distance linked in $G$ and $\left(G_{1}-x y\right) \subseteq G$, it follows that $\{u, v\}$ is also globally distance linked in $G$.

The core of a 2-connected mixed graph $G=(V ; D, E), C(G)$, is defined to be the maximal subset of $V$ in which no pair of vertices is separated by a direction unbalanced 2-separation of $G$. It can be seen that the core of $G$ is unique and can be obtained by the following recursive procedure. If $G$ is direction balanced then put $C(G)=V$. Otherwise choose a 2-separation $\left(H_{1}, H_{2}\right)$ with $V\left(H_{1}\right) \cap V\left(H_{2}\right)=\{a, b\}$ and $H_{2}$ length pure. Let $G_{1}$ be obtained from $H_{1}$ by adding a new length edge $a b$ and put $C(G)=C\left(G_{1}\right)$. For example, the core of the mixed graph in Figure 5 is the set of vertices on the mixed side of its unique 2-separation. A globally rigid cluster of $G$ is a maximal subset of $V$ in which all pairs of vertices are globally linked in $G$. Globally linked pairs and globally rigid clusters in a mixed circuit are determined by its core:

Theorem 5.4 Let $G=(V ; D, L)$ be a mixed circuit and let $C$ be its core. Then

(a) a pair $\{u, v\} \subseteq V$ is globally linked in $G$ if and only if $\{u, v\} \subseteq C$,

(b) $C$ is the only globally rigid cluster of $G$.

Proof: Since (b) follows immediately from (a) we need only prove (a).

We first suppose that $\{u, v\} \nsubseteq C$. Then there exists a direction unbalanced 2-separation $\left(H_{1}, H_{2}\right)$ of $G$ with $V\left(H_{1}\right) \cap V\left(H_{2}\right)=\{a, b\}, H_{2}$ length pure, and $\{u, v\} \nsubseteq V\left(H_{1}\right)$. Let $(G, p)$ be a generic realization of $G$ and $(G, q)$ be the equivalent realization obtained from $(G, p)$ by reflecting $\left(H_{2},\left.p\right|_{H_{2}}\right)$ in the line through $p(a)$ and $p(b)$. Then $l_{p}(u, v) \neq l_{q}(u, v)$ or $s_{p}(u, v) \neq s_{q}(u, v)$ (or both), so $\{u, v\}$ is not globally linked in $G$.

We next suppose that $\{u, v\} \subseteq C$. If $G$ is direction balanced then $G$ is globally rigid by Theorem 1.6, so we may assume that $G$ has a direction unbalanced 2-separation $\left(H_{1}, H_{2}\right)$ with $V\left(H_{1}\right) \cap V\left(H_{2}\right)=\{a, b\}$ and $H_{2}$ length pure. Since $\{u, v\} \subseteq C$ we have $\{u, v\} \subseteq V\left(H_{1}\right)$. Let $G_{1}$ be obtained from $H_{1}$ by adding a new length edge $a b$. By Lemma 5.1(b), $G_{1}$ is a mixed circuit. Furthermore, $G_{1}$ has the same core as $G$. By induction $\{u, v\}$ is globally linked in $G_{1}$. Since $\{a, b\}$ is globally distance linked in $G$ by Lemma 5.3 and 
$G_{1}-a b \subseteq G,\{u, v\}$ is globally linked in $G$.

Note that, in the analogous result to Theorem 5.4(b) for length frameworks given in [9], a graph $G=(V, E)$ may contain several globally rigid clusters and the union of these clusters is equal to $V$.

By using similar techniques to [9] it is also possible to characterize 'uniquely localizable vertices' in a mixed circuit, with respect to a given set $P \subseteq V$ of 'pinned' vertices. We can also determine the number of noncongruent generic realizations of an arbitrary mixed circuit.

\section{Concluding remarks}

We have shown that, under certain conditions, mixed versions of the Henneberg operations preserve global mixed rigidity. We use these results in [10], together with new inductive constructions, to give a characterization for globally rigid mixed circuits, see Theorem 1.6. The characterization of globally length rigid graphs was first given in [2] for the special case when the edge set of the graph is a circuit in the length rigidity matroid. The complete characterization was then obtained in [8] by extending the result to ' $M$-connected graphs', i.e. to graphs with a connected length rigidity matroid. It is conceivable that a similar approach could be used to show that an $M$-connected mixed graph is globally rigid if and only if it is direction balanced. Such a result would not give a complete characterization, however, because $M$-connectivity is not a necessary condition for global rigidity in mixed graphs. This can be seen by considering, for example, any minimally rigid mixed graph with exactly one length edge. Characterizing global rigidity in mixed graphs which are not $M$-connected appears to be a more difficult open problem.

\section{References}

[1] L. Asimow and B. Roth, The rigidity of graphs, Trans. Amer. Math. Soc., Ser. B., 245 (1978) 279-289.

[2] A.R. Berg And T. Jordán, A proof of Connelly's conjecture on 3-connected circuits of the rigidity matroid, J. Combinatorial Theory, Ser. B., Vol. 88, 7797, 2003.

[3] R. Connelly, Generic global rigidity, Discrete Comput. Geom. 33 (2005), no. $4,549-563$. 
[4] T. Eren, W. Whiteley, A.S. Morse, P.N. Belhumeur, And B.D.O. AndERson, Sensor and network topologies of formations with direction, bearing and angle information between agents, In Proc. of the 42nd IEEE Conference on Decision and Control, pp. 3064-3069, 2003.

[5] M. D. Fried And M. Jarden, Field Arithmetic, Results in Mathematics and Related Areas (3), 11. Springer-Verlag, Berlin, 1986.

[6] B. Hendrickson, Conditions for unique graph realizations, SIAM J. Comput. 21 (1992), no. 1, 65-84.

[7] L. Henneberg, Die Graphische Statik der starren Systeme, 1911.

[8] B. JaCKson And T. Jordán, Connected rigidity matroids and unique realizations of graphs, J. Combinatorial Theory Ser B, Vol. 94, 1-29, 2005.

[9] B. Jackson, T. Jordán, And Z. SzabadkA, Globally linked pairs of vertices in equivalent realizations of graphs, Discrete and Computational Geometry, Vol. 35, 493-512, 2006.

[10] B. JaCKSON AND T. Jordán, Globally rigid circuits of the direction-length rigidity matroid, J. Combinatorial Theory Ser B, in press.

[11] L. Lovász And Y. Yemini, On generic rigidity in the plane, SIAM J. Algebraic Discrete Methods 3 (1982), no. 1, 91-98.

[12] J.W. MiLnor, Topology from the differentiable viewpoint, University Press of Virginia, Charlottesville, 1965.

[13] J.B. SAxE, Embeddability of weighted graphs in $k$-space is strongly NPhard, Tech. Report, Computer Science Department, Carnegie-Mellon University, Pittsburgh, PA, 1979.

[14] B. Servatius and W. Whiteley, Constraining plane configurations in CAD: Combinatorics of directions and lengths, SIAM J. Discrete Math., 12, (1999) $136-153$.

[15] I. Streinu, Parallel-redrawing mechanisms, pseudo-triangulations and kinetic planar graphs, P. Healy and N.S. Nikolov (Eds.): GD 2005, Springer LNCS 3843, pp. 421-433, 2005.

[16] T-S. TAY And W. Whiteley, Generating isostatic frameworks, Structural Topology No. 11 (1985), 21-69.

[17] W. Whiteley, Some matroids from discrete applied geometry. Matroid theory (Seattle, WA, 1995), 171-311, Contemp. Math., 197, Amer. Math. Soc., Providence, RI, 1996.

[18] W. Whiteley, Rigidity of molecular structures: geometric and generic analysis, in: Rigidity theory and applications (Edited by M.F. Thorpe and P.M. Duxbury), Kluwer 1999, pp. 21-46. 


\section{A Proof of Theorem 2.1}

We will need some basic concepts and results from differential topology. Let $U$ be an open subset of $\mathbb{R}^{m}$ and $f: U \rightarrow \mathbb{R}^{n}$ be a smooth map. For $X \subset \mathbb{R}^{n}$ let $f^{-1}(X)=\{u \in U: f(u) \in X\}$. Let $k$ be the maximum rank of the Jacobian $\left.d f\right|_{u}$ over all $u \in U$. A point $u \in U$ is a regular point of $f$ if rank $\left.d f\right|_{u}=k$, and $f(u)$ is a regular value of $f$ if $f^{-1}(f(u))$ contains only regular points. If $f(u)$ is a regular value of $f$, then $f^{-1}(f(u))$ is an $(m-k)$-dimensional manifold (see [12, Lemma 1, page 11]).

We will use the rigidity matrix $R(G, p)$ and the rigidity map $f_{G}$ associated to a mixed graph $G=(V ; D, L)$ as defined in Sections 2 and 3 . We will also use the observations made in these sections that rank $R(G, p)$ remains unchanged if we delete the two columns indexed by any vertex of $G$, and that rank $\left.d f_{G}\right|_{p}=\operatorname{rank} R(G, p)$.

Lemma A.1 Let $G=(V ; D, L)$ be a mixed graph and $(G, p)$ be a realization of $G$ such that its rigidity matrix $R(G, p)$ has maximum rank over all realizations of $G$. Then $(G, p)$ is rigid if and only if $(G, p)$ is infinitesimally rigid.

Proof: Let $V=\left\{v_{1}, v_{2}, \ldots, v_{n}\right\}$. If $p\left(v_{i}\right)=p\left(v_{j}\right)$ for some direction edge $e=v_{i} v_{j}$ then $e$ would have no effect on the rigidity or global rigidity of $(G, p)$ so could be deleted. Hence we may suppose that this does not occur. We may now choose our coordinate system such that $(G, p)$ has no vertical direction edges and has $p\left(v_{1}\right)=(0,0)$. Let $T$ be the set of all points $q \in \mathbb{R}^{2 n}$ such that $(G, q)$ has no vertical direction edges and has $q\left(v_{1}\right)=(0,0)$. For each $q=\left(0,0, q_{3}, q_{4}, \ldots, q_{2 n}\right) \in T$ let $\hat{q}=\left(q_{3}, q_{4}, \ldots, q_{2 n}\right)$ and put $\hat{T}=\{\hat{q}$ : $q \in T\}$. Then $\hat{T}$ is an open subset of $\mathbb{R}^{2 n-2}$. Define $f: \hat{T} \rightarrow \mathbb{R}^{|D|+|L|}$ by $f(\hat{q})=f_{G}(q)$ and let $k=\left.\operatorname{rank} d f\right|_{\hat{p}}$. We have $\left.\operatorname{rank} d f_{G}\right|_{q}=\operatorname{rank} R(G, q)=$ $\left.d f\right|_{\hat{q}}$ for all $q \in T$ since $\operatorname{rank} R(G, q)$ remains unchanged if we delete the two columns indexed by $v_{1}$. Hence $\hat{p}$ is a regular value of $f$. By continuity, there exists an open neighbourhood $W \subset \hat{T}$ of $\hat{p}$ such that rank $\left.d f\right|_{\hat{w}}=k$ for all $w \in W$. Let $g=\left.f\right|_{W}$. Then $f(\hat{p})$ is a regular value of $g$. Hence $M=g^{-1}(g(\hat{p}))=f^{-1}(f(\hat{p})) \cap W$ is a $(2 n-2-k)$-dimensional manifold.

Suppose $(G, p)$ is not infinitesimally rigid. Then $k=\operatorname{rank} R(G, p)<$ $2 n-2$ and hence $M$ has dimension at least one. Thus we may choose a sequence of points $\hat{p}_{i} \in M-\{\hat{p}\}$, converging to $\hat{p}$. Since $\hat{p}_{i} \in M, f\left(\hat{p}_{i}\right)=f(p)$ and hence $\left(G, p_{i}\right)$ is equivalent to $(G, p)$. Since $p_{i}(v)=(0,0)=p(v)$ and $\hat{p}_{i} \neq \hat{p},\left(G, p_{i}\right)$ is not a translation of $(G, p)$. Furthermore, when $\hat{p}_{i}$ is close enough to $\hat{p},\left(G, p_{i}\right)$ is not a dilation of $(G, p)$ by -1 . Hence $\left(G, p_{i}\right)$ is not 
congruent to $(G, p)$ whenever $p_{i}$ is close enough to $p$. Thus $(G, p)$ is not rigid.

On the other hand, if $(G, p)$ is infinitesimally rigid then $M$ is a 0 dimensional manifold and hence is a collection of discrete points in $\mathbb{R}^{2 n-2}$. Hence there exists an $\epsilon>0$ such that $f(\hat{q}) \neq f(\hat{p})$ whenever $q \in \hat{T}$ and $\|\hat{p}-\hat{q}\|<\epsilon$. This implies that $(G, p)$ is rigid.

Lemma A.1 immediately implies Theorem 2.1 since rank $R(G, p)$ is maximized in both the cases when $(G, p)$ is infinitesimally rigid and when it is generic.

\section{B Proofs of Lemmas 3.2 and 3.3}

A point $x \in \mathbb{R}^{n}$ is generic if its coordinates form an algebraically independent set over $\mathbb{Q}$.

Lemma B.1 Let $f_{i}$ and $g_{i}$ be non-zero polynomials with integer coefficients in the indeterminates $x_{1}, x_{2}, \ldots, x_{n}$, and $r_{i}=f_{i} / g_{i}$ for $1 \leq i \leq m$. Let $T_{i}=$ $\left\{x \in \mathbb{R}^{n}: g_{i}(x) \neq 0\right\}$ for $1 \leq i \leq m$ and put $T=\bigcap_{i=1}^{m} T_{i}$. Let $f: T \rightarrow \mathbb{R}^{m}$ by $f(x)=\left(r_{1}(x), r_{2}(x), \ldots, r_{m}(x)\right)$. Suppose that $\max _{x \in \mathbb{R}^{n}}\left\{\right.$ rank $\left.\left.d f\right|_{x}\right\}=m$. If $p$ is a generic point in $\mathbb{R}^{n}$, then $p \in T$ and $f(p)$ is a generic point in $\mathbb{R}^{m}$.

Proof: Since $p$ is generic, we have $p \in T$ and rank $\left.d f\right|_{p}=m$. Relabelling if necessary, we may suppose that the first $m$ columns of $\left.d f\right|_{p}$ are linearly independent. Let $p=\left(p_{1}, p_{2}, \ldots, p_{n}\right)$. Define $f^{\prime}: \mathbb{R}^{m} \rightarrow \mathbb{R}^{m}$ by $f^{\prime}\left(x_{1}, x_{2}, \ldots, x_{m}\right)=f\left(x_{1}, x_{2}, \ldots, x_{m}, p_{m+1}, \ldots, p_{n}\right)$. Let $p^{\prime}=\left(p_{1}, p_{2}, \ldots, p_{m}\right)$. Then $f^{\prime}\left(p^{\prime}\right)=f(p)$ and rank $\left.d f^{\prime}\right|_{p^{\prime}}=m$.

Let $f^{\prime}\left(p^{\prime}\right)=\left(\beta_{1}, \beta_{2}, \ldots, \beta_{m}\right)$. Suppose that $h\left(\beta_{1}, \beta_{2}, \ldots, \beta_{m}\right)=0$ for some polynomial $h$ with integer coefficients. Then $h\left(r_{1}(p), r_{2}(p), \ldots, r_{m}(p)\right)=$ 0 . Since $p$ is generic, we must have $h\left(f^{\prime}(x)\right)=0$ for all $x \in \mathbb{R}^{m}$. By the inverse function theorem $f^{\prime}$ maps a sufficiently small open neighbourhood $U$ of $p^{\prime}$ diffeomorphically onto $f^{\prime}(U)$. Thus $h(y)=h\left(f^{\prime}(x)\right)=0$ for all $y \in f^{\prime}(U)$. Since $h$ is a polynomial map and $f^{\prime}(U)$ is an open subset of $\mathbb{R}^{m}$, we have $h=0$. Hence $f^{\prime}\left(p^{\prime}\right)=f(p)$ is generic.

Lemma B.2 Let $f_{i}$ and $g_{i}$ be non-zero polynomials with integer coefficients in the indeterminates $x_{1}, x_{2}, \ldots, x_{n}$ and $r_{i}=f_{i} / g_{i}$ for $1 \leq i \leq n$. Let $T_{i}=\left\{x \in \mathbb{R}^{n}: g_{i}(x) \neq 0\right\}$ for $1 \leq i \leq n$ and put $T=\bigcap_{i=1}^{n} T_{i}$. Let $f: T \rightarrow \mathbb{R}^{n}$ by $f(x)=\left(r_{1}(x), r_{2}(x), \ldots, r_{n}(x)\right)$. Suppose that $f(p)$ is a 
generic point in $\mathbb{R}^{n}$ for some $p \in T$. Let $L=\mathbb{Q}(p)$ and $K=\mathbb{Q}(f(p))$. Then $\tilde{K}=\tilde{L}$.

Proof: Since $r_{i}(x)$ is a ratio of two polynomials with integer coefficients, we have $r_{i}(p) \in L$ for all $1 \leq i \leq n$. Thus $K \subseteq L$. Since $f(p)$ is generic, by Lemma B.1 we have $t d[K: \mathbb{Q}]=n$. Since $K \subseteq L$ and $L=\mathbb{Q}(p)$ we have $t d[L: \mathbb{Q}]=n$. Thus $\tilde{K} \subseteq \tilde{L}$ and $t d[\tilde{K}: \mathbb{Q}]=n=t d[\tilde{L}: \mathbb{Q}]$. Suppose $\tilde{K} \neq \tilde{L}$, and choose $\gamma \in \overline{\tilde{L}}-\tilde{K}$. Then $\gamma$ is not algebraic over $K$ so $S=\left\{\gamma, f_{1}(p), f_{2}(p), \ldots, f_{n}(p)\right\}$ is algebraically independent over $\mathbb{Q}$. This contradicts the facts that $S \subseteq \tilde{L}$ and $t d[\tilde{L}: \mathbb{Q}]=n$.

Proof of Lemma 3.2: Choose a generic framework $(G, q)$ conguent to $(G, p)$. Since $G$ is independent, rank $\left.d f_{G}\right|_{q}=|D|+|L|$. Hence Lemma B.1 implies that $f_{G}(q)$ is generic. The lemma now follows since $f_{G}(p)=f_{G}(q)$.

Proof of Lemma 3.3: Let the vertices of $G$ be $v_{1}, v_{2}, \ldots, v_{n}$. Let $T$ be the set of all points $q \in \mathbb{R}^{2 n}$ such that $(G, q)$ has no vertical direction edges and $q\left(v_{1}\right)=(0,0)$. For each $q=\left(0,0, q_{3}, q_{4}, \ldots, q_{2 n}\right) \in T$ let $\hat{q}=\left(q_{3}, q_{4}, \ldots, q_{2 n}\right)$ and put $\hat{T}=\{\hat{q}: q \in T\}$. Define $f: \hat{T} \rightarrow \mathbb{R}^{2 n-2}$ by

$$
f\left(q_{3}, q_{4}, \ldots, q_{2 n}\right)=f_{G}\left(0,0, q_{3}, q_{4}, \ldots, q_{2 n}\right) .
$$

Let $\hat{p}=\left(p_{3}, p_{4}, \ldots, p_{2 n}\right)$. Then $f(\hat{p})=f_{G}(p)$ is generic by hypothesis. We have $L=\mathbb{Q}(\hat{p})$ and $K=\mathbb{Q}(f(\hat{p}))$. By Lemma B.2, $\tilde{K}=\tilde{L}$. Furthermore, $2 n-2=t d[\tilde{K}, \mathbb{Q}]=t d[\tilde{L}, \mathbb{Q}]$. Thus $\hat{p}$ is a generic point in $\mathbb{R}^{2 n-2}$. 